This item was submitted to Loughborough's Research Repository by the author.

Items in Figshare are protected by copyright, with all rights reserved, unless otherwise indicated.

\title{
Decay of correlation for random intermittent maps
}

PLEASE CITE THE PUBLISHED VERSION

http://dx.doi.org/10.1088/0951-7715/27/7/1543

\section{PUBLISHER}

(c) IOP Publishing Ltd \& London Mathematical Society

\section{VERSION}

AM (Accepted Manuscript)

\section{PUBLISHER STATEMENT}

This work is made available according to the conditions of the Creative Commons Attribution-NonCommercialNoDerivatives 4.0 International (CC BY-NC-ND 4.0) licence. Full details of this licence are available at: https://creativecommons.org/licenses/by-nc-nd/4.0/

\section{LICENCE}

CC BY-NC-ND 4.0

\section{REPOSITORY RECORD}

Bahsoun, Wael, Christopher Bose, and Yuejiao Duan. 2019. "Decay of Correlation for Random Intermittent Maps". figshare. https://hdl.handle.net/2134/23279. 


\title{
DECAY OF CORRELATION FOR RANDOM INTERMITTENT MAPS
}

\author{
WAEL BAHSOUN ${ }^{\dagger}$, CHRISTOPHER BOSE* $^{*}$, AND YUEJIAO DUAN ${ }^{\ddagger}$
}

\begin{abstract}
We study a class of random transformations built over finitely many intermittent maps sharing a common indifferent fixed point. Using a Young-tower technique, we show that the map with the fastest relaxation rate dominates the asymptotics. In particular, we prove that the rate of correlation decay for the annealed dynamics of the random map is the same as the sharp rate of correlation decay for the map with the fastest relaxation rate.
\end{abstract}

\section{INTRODUCTION}

General statistical properties of deterministic expanding maps of the interval with a neutral fixed point are by now well understood. In [12] Pianigiani proved existence of invariant densities of such maps. In $[7,8,14]$ it was independently proved that such maps exhibit a polynomial rate of correlation decay. Later Gouëzel [4] showed the rate obtained in [14] is in fact sharp. The slow mixing behaviour of such maps made them a useful testing ground for physical problems with intermittent behaviour: systems whose orbits spend very long time in a certain small part of the phase space.

In this paper we are interested in studying i.i.d. randomized compositions of two intermittent maps sharing a common indifferent fixed point. It is intuitively clear that the annealed ${ }^{1}$ dynamics of the random process will also have a polynomial rate of correlation decay. However, we are interested in the following question: How do the asymptotics of the random map relate to those of the original maps; in particular, the rate of correlation decay?

We show that the map with the fast relaxation rate dominates the asymptotics (see Theorem 2.3 for a precise statement). Interestingly, in our setting, the map with slow relaxation rate is allowed to be of 'boundary-type', and consequently admit an infinite ( $\sigma$-finite) invariant measure, but the random system will always admit an absolutely continuous invariant probability measure. We obtain our result by using

Date: November 27, 2015.

1991 Mathematics Subject Classification. Primary 37A05, 37E05.

Key words and phrases. Interval maps with a neutral fixed point, intermittency, random dynamical systems, decay of correlations.

${ }^{1}$ Annealed dynamics refers to the randomized dynamics, averaged over the randomizing space, see Subsection 2.2 and Theorem 2.3. This should be contrasted with the notion of quenched dynamics, the behaviour of the system with one random choice of the randomizing sequence. The term almost sure dynamics is also used to refer to quenched dynamics. 
a version of the skew product representation ${ }^{2}$ studied in [2] and a Young-tower technique [14].

In Section 2 we introduce our random system and its skew product representation. The statement of our main result Theorem 2.3 is also in Section 2. In Section 3 we build a Young-tower for the skew product representation. Proofs, including the proof of Theorem 2.3, are in Section 4.

\section{Setup and Statement of the main Result}

2.1. A random dynamical system. Let $(I, \mathfrak{B}(I), m)$ be the measure space, with $I=[0,1], \mathfrak{B}(I)$ the Borel $\sigma$-algebra and $m$ being Lebesgue measure. By a LiveraniSaussol-Vaienti (LSV)-map we mean a member of the parameterized family of maps on $I$ given by

$$
T_{\alpha}(x)=\left\{\begin{array}{ll}
x\left(1+2^{\alpha} x^{\alpha}\right) & x \in\left[0, \frac{1}{2}\right] \\
2 x-1 & x \in\left(\frac{1}{2}, 1\right]
\end{array} .\right.
$$

Here the parameter $\alpha \in(0, \infty)$. Each LSV map has a neutral fixed point at $x=0$. For $0<\alpha<1, T_{\alpha}$ admits a finite, absolutely continuous invariant measure while for $\alpha \geq 1$ the absolutely continuous invariant measure is $\sigma$ - finite. See [12] and [13] for the some of the earliest results of this type.

Let $0<\alpha_{1}<\alpha_{2}<\cdots<\alpha_{r} \leq 1$. We consider a random map $T$ which is given by:

$$
T(x):=\left\{T_{\alpha_{1}}(x), T_{\alpha_{2}}(x), \ldots T_{\alpha_{r}}(x) ; p_{1}, p_{2}, \ldots p_{r}\right\},
$$

where $p_{i}>0$ and $\sum p_{i}=1$. Note that all the individual maps share a single common neutral fixed point at $x=0$.

Assumption 2.1. Since nothing we will do in the sequel depends on $r$, the number of maps making up the random map, we will restrict the discussion to the case $r=2$ and denote the parameters

$$
0<\alpha<\beta \leq 1 .
$$

At the same time, this will simplify our notation:

$$
T(x):=\left\{T_{\alpha}(x), T_{\beta}(x) ; p_{1}, p_{2}\right\} .
$$

The random map $T$ in (2.3) maybe viewed as a Markov process with transition function

$$
\mathbb{P}(x, A)=p_{1} \mathbf{1}_{A}\left(T_{\alpha}(x)\right)+p_{2} \mathbf{1}_{A}\left(T_{\beta}(x)\right)
$$

of a point $x \in I$ into a set $A \in \mathfrak{B}(I)$. The transition function induces an operator, $E_{T}$, acting on measures; i.e., if $\mu$ is a measure on $(I, \mathfrak{B})$,

$$
\left(E_{T} \mu\right)(A)=p_{1} \mu\left(T_{\alpha}^{-1}(A)\right)+p_{2} \mu\left(T_{\beta}^{-1}(A)\right) .
$$

A measure $\mu$ is said to be $T$-invariant if

$$
\mu=E_{T} \mu,
$$

and $\mu$ is said to be an absolutely continuous invariant measure if $d \mu=f^{*} d m$, $\int_{I} f^{*} d m=1$. To study absolutely continuous invariant measures, we introduce the transfer operator (Perron-Frobenius) of the random map $T$ :

$$
\left(P_{T} f\right)(x)=p_{1} P_{T_{\alpha}}(f)(x)+p_{2} P_{T_{\beta}}(f)(x),
$$

\footnotetext{
${ }^{2}$ The skew product studied in this paper is also related to a skew product studied by Gouëzel in [5]. See Remark 2.6 for more details.
} 
where $P_{T_{\alpha}}, P_{T_{\beta}}$ denote the transfer operators associated with the $T_{\alpha}, T_{\beta}$ respectively. Then it is a straight-forward computation to show that a measure $\mu=f^{*} \cdot m$ is absolutely continuous invariant measure if

$$
P_{T} f^{*}=f^{*} .
$$

2.2. Skew product representation. By the annealed dynamics of the random map we mean the statistics of the random dynamical system averaged over the randomizing space (see [1] for a general treatment of annealed versus quenched interpretation). Probabilistic aspects of $T$, in particular the correlation decay of the annealed dynamics, are frequently studied by constructing a Young-tower for the skew-product representation of $T$. For this purpose, we are going to use a version $^{3}$ of the skew product representation which was studied in [2]. Define the skew product transformation $S(x, \omega): I \times I \rightarrow I \times I$ by

$$
S(x, \omega)=\left(T_{\alpha(\omega)}, \varphi(\omega)\right),
$$

where

$$
\alpha(\omega)=\left\{\begin{array}{ll}
\alpha & , \omega \in\left[0, p_{1}\right) \\
\beta & , \omega \in\left[p_{1}, 1\right]
\end{array} \quad ; \quad \varphi(\omega)=\left\{\begin{array}{ll}
\frac{\omega}{p_{1}} & , \omega \in\left[0, p_{1}\right) \\
\frac{\omega-p_{1}}{p_{2}} & , \omega \in\left[p_{1}, 1\right]
\end{array} .\right.\right.
$$

We denote the transfer operator associated with $S$ by $\mathcal{L}_{S}$ : for $g \in L^{1}(I \times I)$ and measurable $A \subseteq I \times I$,

$$
\int_{S^{-1} A} g d(m \times m)(x, \omega)=\int_{A} \mathcal{L}_{S} g d(m \times m)(x, \omega) .
$$

Then a measure $\nu$, such that $d \nu=g^{*} d(m \times m)$ and $\int_{I \times I} g^{*} d(m \times m)=1$, is an absolutely continuous $S$-invariant measure if

$$
\mathcal{L}_{S} g^{*}=g^{*} .
$$

In [2], Theorem 5.2 it is shown that if $g \in L^{1}(I \times I)$ and $\mathcal{L}_{S} g=\lambda g$ with $|\lambda|=1$, then

$$
g(x, \omega)=f(x) \cdot \mathbf{1}(\omega)
$$

and $P_{T} f=\lambda f$, that is, $g$ depends only on the spatial coordinate $x$ and as a function of $x$ only, is also an eigenfunction for $P_{T}$. Setting $\lambda=1$ we obtain $\mathcal{L}_{S} g^{*}=g^{*}$ if and only if $g^{*}(x, \omega)=f^{*}(x)$ with $P_{T} f^{*}=f^{*}$. Consequently there is a one to one correspondence between invariant densities for $S$ and invariant densities for $T$. Moreover, dynamical properties such as ergodicity, number of ergodic components or weak-mixing, properties that are determined by peripheral eigenfunctions, can be determined via either system.

On the other hand, properties like correlation decay (or even strong mixing) cannot be established by peripheral spectrum alone.

Definition 2.2. Suppose $\tau: X \rightarrow X$ preserves the measure $\mu$ on $X$. For $f \in$ $L^{\infty}(X, \mu)$ and $g \in L^{1}(X, \mu)$ denote by

$$
\operatorname{Cor}_{n}(f, g)=\operatorname{Cor}_{n, \tau}(f, g):=\int_{X} f \circ \tau^{n} \cdot g d \mu-\int_{X} f d \mu \int_{X} g d \mu .
$$

\footnotetext{
${ }^{3}$ The results obtained in [2] are valid for any class of measurable non-singular maps on $\mathbb{R}^{q}$, without any regularity assumptions. Moreover in [2], the probability distribution on the noise space is allowed to be place-dependent.
} 
Normally we will simply write $\operatorname{Cor}_{n}(f, g)$ when the map being applied is understood. Estimates on correlation decay are known in many dynamical settings. For example, it was shown in [14] that for $f \in L^{\infty}, g$ Hölder continuous on $I$ and $\tau=T_{\alpha}, 0<$ $\alpha<1$ an LSV-map, $\left|\operatorname{Cor}_{n, T_{\alpha}}(f, g)\right|=O\left(n^{1-\frac{1}{\alpha}}\right)$. Gouëzel in [4] proved that this rate is sharp.

Our main result, Theorem 2.3, establishes exactly the same rate of correlation decay for the random map.

\subsection{Statement of the main result.}

Theorem 2.3. Let $0<\alpha<\beta \leq 1$ and $S$ be as defined in Subsection 2.2. Then

(1) $S$ admits a unique absolutely continuous invariant probability measure $\nu$;

(2) $(S, \nu)$ is mixing;

(3) for $\phi \in L^{\infty}(I \times I, m \times m)$ and $\psi$ a Hölder continuous function on $I \times I$ we have

$$
\left|\operatorname{Cor}_{n, S}(\phi, \psi)\right|=\mathcal{O}\left(n^{1-\frac{1}{\alpha}}\right) ;
$$

Remark 2.4. Our main goal in Theorem 2.3 is not so much to show that $S$ has polynomial rate of correlation decay, but to discover how the correlation decay for $S$ relates to those of the original maps. Indeed, if $0<\alpha<\beta<1$, and without any further conditions on $\alpha$ and $\beta$, one can easily obtain, by just using the rough estimates contained in Lemma 4.4 and the Young tower construction detailed in the next two sections, an upper bound on the rate of order $\mathcal{O}\left(n^{1-\frac{1}{\beta}}\right)$; that is, the rate of decay is at least as fast as the slowest escape rate map. What we have shown in Theorem 2.3 is that the actual decay rate of the random map is completely determined by the faster escape rate of the map $T_{\alpha}$.

Remark 2.5. It is worth noting that in Theorem $2.3, \beta \leq 1$. The case when $\beta=1$ is interesting on its own since in this case the map $T_{\beta}$ admits only an infinite ( $\sigma$-finite) absolutely continuous invariant measure, but Theorem 2.3 shows that the skew product $S$, and hence the random map $T$, admits a unique absolutely continuous invariant probability measure.

Remark 2.6. Limit theorems for the following related skew product were studied by Gouëzel in [5]:

$$
S(x, \omega)=\left(T_{\alpha(\omega)}, 4 \omega\right)
$$

with $\omega \in \mathbb{S}^{1}$ and $T_{\alpha}(\omega)$ being a random choice of LSV-map from Equation 2.1. For the randomizing process, it is further assumed that

(1) $\alpha(\omega)$ is $C^{2}$;

(2) $0<\alpha_{\min }<\alpha_{\max }<1$;

(3) $\alpha(\omega)$ takes the value $\alpha_{\min }$ at a unique point $\omega_{0} \in \mathbb{S}^{1}$, with $\alpha^{\prime \prime}\left(\omega_{0}\right)>0$;

(4) $\alpha_{\max }<\frac{3}{2} \alpha_{\min }$.

Under the above conditions, using a result of Pène [11] (see [5] Theorem B.1), Gouëzel ([5], Theorem 4.1) obtained asymptotics that would lead to a correlation decay rate of order $\mathcal{O}\left(\sqrt{\log n} \cdot n^{1-\frac{1}{\alpha_{\min }}}\right)$.

Gouëzel remarks in [5] that in his setting the conditions $\alpha_{\max }<1$ and $\alpha_{\max }<\frac{3}{2} \alpha_{\min }$ may be technical artifacts, arising from the method of proof.

Remark 2.7. In our i.i.d. setting, we rely on relatively simple (and classical) estimates on large deviations for i.i.d. randomizers and show, for all $0<\alpha<\beta \leq 1$, 
that the correlation decay rate of the annealed dynamics is exactly the same as the correlation decay rate of the fastest mixing map $T_{\alpha}$. Note that the constraint $\beta \leq 1$ still may be a technical artifact, a consequence of our use of the negative Schwarzian derivative and Koebe Principle in Lemma 4.8, rather than some underlaying obstruction. Indeed, the probabilistic analysis of our model (see Proposition 4.1 ) is valid for the full range of parameter values $0<\alpha<\beta<\infty$, so it may be possible to extend our results to the case of $0<\alpha<1<\beta$ for the (presumably) finite invariant measure case or even $1 \leq \alpha<\beta<\infty$, by following the techniques of $[10]$, where one expects the invariant measure to be only $\sigma$-finite.

\section{A Young-TOWER For $S$}

\subsection{Notation. Set}

$$
T_{\omega}^{n}(x):=T_{\alpha\left(\varphi^{n-1} \omega\right)} \circ \ldots \circ T_{\alpha(\varphi \omega)} \circ T_{\alpha(\omega)}(x) .
$$

Then

Also, set

$$
S^{n}(x, \omega)=\left(T_{\omega}^{n}(x), \varphi^{n}(\omega)\right)
$$

$$
P_{\omega}^{n}:=p_{\alpha\left(\varphi^{n-1} \omega\right)} \times \ldots \times p_{\alpha(\varphi \omega)} \times p_{\alpha(\omega)}
$$

where $p_{\alpha(\omega)}=p_{1}$, for $\alpha(\omega)=\alpha$ and $p_{\alpha(\omega)}=p_{2}$, for $\alpha(\omega)=\beta$. We define two sequences of random points $\left\{x_{n}(\omega)\right\}$ and $\left\{x_{n}^{\prime}(\omega)\right\}$ in $[0,1]$ which will be useful in the construction of a suitable Young tower. The points $x_{n}(\omega)$ lie in $(0,1 / 2]$. Set

$$
x_{1}(\omega) \equiv \frac{1}{2} \text { and } x_{n}(\omega)=\left.T_{\alpha(\omega)}^{-1}\right|_{\left[0, \frac{1}{2}\right]}\left[x_{n-1}(\varphi \omega)\right], n \geq 2 .
$$

Observe that with this notation,

$$
S\left(x_{n}(\omega), \omega\right)=\left(T_{\alpha(\omega)}\left(x_{n}(\omega)\right), \varphi \omega\right)=\left(x_{n-1}(\varphi \omega), \varphi \omega\right) .
$$

The points $\left\{x_{n}^{\prime}(\omega)\right\}$ lie in $\left(\frac{1}{2}, 1\right]$, defined by

$$
x_{0}^{\prime}(\omega) \equiv 1, x_{1}^{\prime}(\omega) \equiv \frac{3}{4} \text { and } x_{n}^{\prime}(\omega)=\frac{x_{n}(\varphi \omega)+1}{2}, n \geq 2,
$$

that is, $\left\{x_{n}^{\prime}(\omega)\right\}$ are preimages of $\left\{x_{n}(\varphi \omega)\right\}$ in $\left(\frac{1}{2}, 1\right]$ under the right branch $2 x-1$.

3.2. A tower for $S$. Let $\Delta_{0}=\left(\frac{1}{2}, 1\right] \times[0,1)$. Let $R: \Delta_{0} \rightarrow \mathbb{Z}^{+}$be the first return time function and $S^{R}: \Delta_{0} \rightarrow \Delta_{0}$ be the return map. $\Delta_{0}$ is referred to as the base of the tower $\Delta$ which is given by

$$
\Delta:=\left\{(z, n): z \in \Delta_{0} \text { and } n=0,1, \ldots, R(z)-1\right\} .
$$

Let $F: \Delta \rightarrow \Delta$ be the map acting on the tower as follows:

$$
F(z, l)= \begin{cases}(z, l+1), & \text { if } l<R(z)-1 \\ \left(S^{R}(z), 0\right), & \text { if } l=R(z)-1\end{cases}
$$

We refer to $\Delta_{l}:=\Delta \cap\{n=l\}$ as the $l^{\text {th }}$ level of the tower. For $n \geq 1$, set $I_{n}(\omega):=\left(x_{n+1}(\omega), x_{n}(\omega)\right]$ and $J_{n}(\omega):=\left(x_{n}^{\prime}(\omega), x_{n-1}^{\prime}(\omega)\right]$. Observe that every point in $J_{n}(\omega)$ will return to $\left(\frac{1}{2}, 1\right]$ in $n$ steps under the random iteration $T_{\omega}^{n}$ as follows:

$$
J_{n}(\omega) \rightarrow I_{n-1}(\varphi \omega) \rightarrow I_{n-2}\left(\varphi^{2} \omega\right) \rightarrow \ldots \rightarrow I_{1}\left(\varphi^{n-1} \omega\right) \rightarrow\left(\frac{1}{2}, 1\right]
$$


Next we partition $\Delta_{0}$, into subsets $\Delta_{0, i}, i=1,2, \ldots$ where

$$
\Delta_{0, i}:=\left\{(x, \omega) \mid x \in J_{i}(\omega)\right\}
$$

and then further partition each $\Delta_{0, i}$ into subsets $\Delta_{0, i}^{j}, j=1,2, \ldots 2^{i}$ according to the $2^{i}$ possible values of the string $\alpha(\omega), \alpha(\varphi \omega), \ldots \alpha\left(\varphi^{i-1} \omega\right)$. Defined this way, $S^{i}$ maps each subset $\Delta_{0, i}^{j}$ bijectively to $\Delta_{0}$.

For example, in the case $i=2$, there are four sets $\Delta_{0,2}^{j}$ on which $R=2$ and such that $S^{R}$ maps each set bijectively to $\Delta_{0}$ :

$$
\Delta_{0,2}^{j}= \begin{cases}J_{2}(\omega) \times\left[0, p_{1}^{2}\right) & , \text { if } j=1, \\ J_{2}(\omega) \times\left[p_{1}^{2}, p_{1}\right) & , \text { if } j=2, \\ J_{2}(\omega) \times\left[p_{1}, p_{1}+p_{1} \cdot p_{2}\right) & , \text { if } j=3, \\ J_{2}(\omega) \times\left[p_{1}+p_{1} \cdot p_{2}, 1\right) & , \text { if } j=4 .\end{cases}
$$

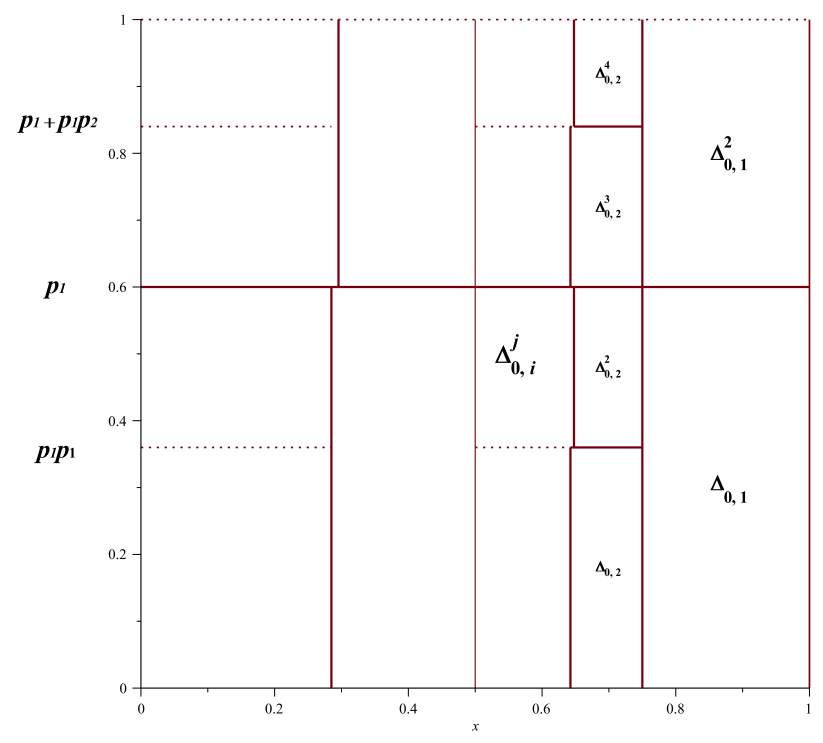

Figure 1. An example of the base of the tower when $\alpha=0.5$ $\beta=0.7, p_{1}=0.6$. 
To summarize,

$$
\Delta_{0, i}=\bigcup_{j=1}^{2^{i}} \Delta_{0, i}^{j} .
$$

and for the base,

$$
\Delta_{0}=\bigcup_{i=1}^{\infty} \bigcup_{j=1}^{2^{i}} \Delta_{0, i}^{j}
$$

where, for every $i$ and $j=1,2, \ldots, 2^{i}$,

$$
\left.R\right|_{\Delta_{0, i}^{j}}=i \text {. }
$$

Finally the tower $\Delta$ is partitioned by

$$
\Delta=\bigcup_{i=1}^{\infty} \bigcup_{l=0}^{i-1}\left(\bigcup_{j=1}^{2^{i}} \Delta_{l, i}^{j}\right)
$$

An example of the partition of the base of the tower and a few images of the partition elements under $S$ is presented in Figure 1.

3.3. Using Young's technique to prove Theorem 2.3. We say $s\left(z_{1}, z_{2}\right)$ is a separation time for $z_{1}, z_{2} \in \Delta_{0}$ if $s$ is the smallest $n \geq 0$ such that $\left(F^{R}\right)^{n}\left(z_{1}\right)$ and $\left(F^{R}\right)^{n}\left(z_{2}\right)$ lie in distinct $\Delta_{0, i}^{j}$. Also let $\hat{R}: \Delta \rightarrow \mathbb{Z}$ be the function defined by

$$
\hat{R}(x, \omega)=\text { the smallest integer } n \geq 0 \text { s.t. } F^{n}(x, \omega) \in \Delta_{0} .
$$

To prove Theorem 2.3, we have to:

(A) Prove that $\int_{I \times I} R d(m \times m)<\infty$, and establish the asymptotic estimate $(m \times m)\{\hat{R}>n\}=\mathcal{O}\left(n^{1-\frac{1}{\alpha}}\right)$,

(B) Establish the bounded distortion conditions on the return map: there exists $0<\theta<1$ and $C(F)>0$ such that

$\left|\frac{D F^{R}\left(z_{1}\right)}{D F^{R}\left(z_{2}\right)}-1\right| \leq C(F) \cdot \theta^{s\left(F^{R}\left(z_{1}\right), F^{R}\left(z_{2}\right)\right)}, \forall i=1,2, \ldots, \forall j=1, \ldots, 2^{i}, \forall z_{1}, z_{2} \in \Delta_{0, i}^{j}$,

(C) Confirm that the return times are aperiodic.

(A) is established by Proposition 4.1 in Subsection 4.1 while (B) is the content of Proposition 4.10 in Subsection 4.2. Since we have all possible integer return times, (C) is immediate. It is interesting to note that the upper bound constraint $\beta \leq 1$ specified in our main result, Theorem 2.3, is only used in Proposition 4.10, so the tower asymptotics detailed in (A) hold for all pairs $0<\alpha<\beta<\infty$.

\section{Proofs}

4.1. Estimates on the return sets. Throughout this section we will adopt the notation $E_{\omega}(\cdot)=\int_{I} \cdot(\omega) d \omega$ for expectation with respect to the randomizing variable. Also, we write $a_{n} \sim b_{n}$ if there is a constant $C>1$ such that $C^{-1} b_{n} \leq a_{n} \leq$ $C b_{n}$ for all $n$. 
Proposition 4.1. For all $0<\alpha<\beta<\infty$ we have

$$
E_{\omega}\left(x_{n}(\omega)\right) \sim n^{-\frac{1}{\alpha}}
$$

$$
E_{\omega}\left(x_{n}^{\prime}(\omega)-\frac{1}{2}\right) \sim n^{-\frac{1}{\alpha}}
$$

$$
m \times m\{\hat{R}>n\} \sim n^{1-\frac{1}{\alpha}} .
$$

Before proving this result, we gather some estimates in a sequence of lemmas.

Lemma 4.2. For all $x \in\left[0, \frac{1}{2}\right] T_{\alpha}(x) \geq T_{\beta}(x)$ with strict inequality on the open interval $\left(0, \frac{1}{2}\right)$.

Proof. This is a straightforward calculation.

Corollary 4.3. For $0 \leq x \leq y<\frac{1}{2}$ we have $T_{\alpha}(y) \geq T_{\beta}(x)$ with strict inequality in either situation: $0<x \leq y<\frac{1}{2}$ or $0 \leq x<y \leq \frac{1}{2}$.

We will estimate the position of $x_{n}(\omega)$ by comparing to the sequence of non-random backwards iterates constructed with only one map; either always choosing $\left.T_{\alpha}\right|_{\left[0, \frac{1}{2}\right]} ^{-1}$ or $\left.T_{\beta}\right|_{\left[0, \frac{1}{2}\right]} ^{-1}$ in place of $\left.T_{\alpha}(\omega)\right|_{\left[0, \frac{1}{2}\right]} ^{-1}$ in equation (3.1). Denote these non-random iterates by $x_{n}^{\alpha}$ and $x_{n}^{\beta}$ respectively. It is immediate from Lemma 4.2 that for every $n, x_{n}^{\alpha} \leq$ $x_{n}^{\beta}$. Furthermore, it is well-known that $x_{n}^{\alpha} \sim n^{-\frac{1}{\alpha}}$ with similar estimates for the parameter $\beta$. (See, for example, estimates at the beginning of Section 6.2 of [14].) We begin with a very rough (but intuitively obvious) estimate on $x_{n}(\omega)$.

Lemma 4.4. For all $n \geq 1$ and for all $\omega$

$$
x_{n}^{\alpha} \leq x_{n}(\omega) \leq x_{n}^{\beta} .
$$

Proof. Suppose, to the contrary, $x_{n}(\omega)<x_{n}^{\alpha}$ for some $n, \omega$. Note that if $\alpha\left(\varphi^{k}(\omega)\right)=$ $\alpha$ for all $k$ then $x_{n}(\omega)=x_{n}^{\alpha}$, contradicting our assumption. Let $k \in\{0,1, \ldots n-1\}$ be smallest integer such that $\alpha\left(\varphi^{k}(\omega)\right)=\beta$. Then $x_{n-k}\left(\varphi^{k}(\omega)\right)<x_{n-k}^{\alpha}$ since $T_{\alpha}^{k}$ is increasing and

$$
x_{n-k-1}\left(\varphi^{k+1}(\omega)\right)=T_{\beta}\left(x_{n-k}\left(\varphi^{k}(\omega)\right)\right)<T_{\alpha}\left(x_{n-k}\left(\varphi^{k}(\omega)\right)=x_{n-k-1}^{\alpha} .\right.
$$

Here we have invoked Corollary 4.3. Iterating this argument for each index where $\alpha\left(\varphi^{j}(\omega)\right)=\beta$ gives

$$
\frac{1}{2}=x_{1}\left(\varphi^{n-1}(\omega)\right)<x_{1}^{\alpha}=\frac{1}{2}
$$

which is again a contradiction. A similar argument shows $x_{n}(\omega) \leq x_{n}^{\beta}$ for all $n, \omega$.

Lemma 4.5. Suppose $n$ is given and $K_{0} \in[0, n-1]$ is fixed. Suppose $\omega \in[0,1]$ is such that

$$
\#\left\{j \in\{0,1, \ldots n-1\} \mid \alpha\left(\varphi^{j}(\omega)\right)=\alpha\right\}>K_{0} .
$$

Then $x_{n}^{\alpha} \leq x_{n}(\omega) \leq x_{\left\lfloor K_{0}\right\rfloor}^{\alpha}$ 
Proof. The left-hand inequality is given by Lemma 4.4. For the other side, suppose $x_{n}(\omega)>x_{\left\lfloor K_{0}\right\rfloor}^{\alpha}$. Consider the following sequence of points

$$
y_{n-j}:= \begin{cases}T_{\alpha}\left(x_{n}\left(\varphi^{j} \omega\right)\right) & \text { if } \alpha\left(\varphi^{j} \omega\right)=\alpha \\ x_{n}\left(\varphi^{j} \omega\right) & \text { if } \alpha\left(\varphi^{j} \omega\right)=\beta\end{cases}
$$

For each $j=0,1, \ldots n-1$ define $K(j):=\#\left\{i \in\{0,1, \ldots j\} \mid \alpha\left(\varphi^{i} \omega\right)=\alpha\right\}$.

For example, $K(0)=0$ or $1, K(j) \leq j+1$ and $K(n-1)>\left\lfloor K_{0}\right\rfloor$ by hypothesis.

By an argument similar to the proof of Lemma 4.4, using $T_{\beta}$ compared to the identity map we have

$$
y_{n-j} \leq x_{n-j}(\omega), \text { for all } j=0,1, \ldots n-1 .
$$

On the other hand, comparing $T_{\alpha}$ to the identity map and applying Lemma 4.2 gives

$$
x_{\left\lfloor K_{0}\right\rfloor-j}^{\alpha}<y_{n-j}, \text { for all } j=0,1, \ldots n-1 .
$$

Pick $j_{0}$ so that $K\left(j_{0}\right)=\left\lfloor K_{0}\right\rfloor-1$. Note that $j_{0}<n-1$. Then

$$
\frac{1}{2}=x_{1}^{\alpha}=x_{\left\lfloor K_{0}\right\rfloor-\left(\left\lfloor K_{0}\right\rfloor-1\right)}^{\alpha}<y_{n-j_{0}} \leq x_{n-j_{0}}(\omega)
$$

which contradicts the hitting time of $x_{n}(\omega)$ to the interval $\left[\frac{1}{2}, 1\right]$.

Pick any $0<p_{0}<p_{1}$, fix $n>1$ and let $K_{0}:=n p_{0}$. There are many standard large deviation estimates for i.i.d. random variables that will ensure that most $\omega$ encounter at least $K_{0}$ instances of $\alpha\left(\varphi^{i} \omega\right)=\alpha$ in their first $n$ iterates. As we are aiming for exponential decay in the tail estimate, we invoke a classical result due to Hoeffding [6] that works especially well for our case of Bernoulli random variables. It is precisely at this point that we avoid generating an upper bound constraint on $\beta$ as was the case in Gouëzel [5]. If instead we were to use the more general estimates from the well-known Berry-Esséen Theorem (e.g. Theorem 1, Section XVI.5 in [3]), for example, we would obtain power law decay in the tail leading to the requirement $\beta<\frac{3}{2} \alpha$ in order to complete the proof.

Lemma 4.6. For every $n \geq 1$

$$
\operatorname{Pr}\left\{\omega \mid \#\left\{j \in\{0,1, \ldots n-1\} \mid \alpha\left(\varphi^{j} \omega\right)=\alpha\right\} \leq K_{0}\right\} \leq \exp \left[-2 n\left(p_{1}-p_{0}\right)^{2}\right] .
$$

Proof. Let $S_{n}$ count the number of times the value $\beta$ occurs in the first $n$ iterates. Observe that

$$
\begin{array}{r}
\operatorname{Pr}\left\{\omega \mid \#\left\{j \in\{0,1, \ldots n-1\} \mid \alpha\left(\varphi^{j} \omega\right)=\alpha\right\} \leq K_{0}\right\} \\
\leq \operatorname{Pr}\left\{\omega \mid \#\left\{j \in\{0,1, \ldots n-1\} \mid \alpha\left(\varphi^{j} \omega\right)=\beta\right\} \geq n-K_{0}\right\}
\end{array}
$$

In Theorem 1 of [6] let $\mu=p_{2}$ and let $t=p_{1}-p_{0}<p_{1}=1-\mu$. Then the bottom probability in equation (4.1) equals

$$
\operatorname{Pr}\left\{S_{n}-\mu n \geq\left(1-p_{0}-p_{2}\right) n\right\}=\operatorname{Pr}\left\{\frac{S_{n}}{n}-\mu \geq t\right\} .
$$

The exponential estimate now follows from (2.3) in Theorem 1 of [6]. 
Proof. (Of Proposition 4.1)

(1) For fixed $n$, with $p_{0}<p_{1}$ as above, let $K_{0}=p_{0} n$. Set

$$
G_{n}=\left\{\omega \mid\left\{\omega \mid \#\left\{j \in\{0,1, \ldots n-1\} \mid \alpha\left(\varphi^{j} \omega\right)=\alpha\right\}>K_{0}\right\} .\right.
$$

Lemma 4.6 estimates $\operatorname{Pr}\left(I \backslash G_{n}\right) \leq \exp \left[-2 n\left(p_{1}-p_{0}\right)^{2}\right]$.

Now

$E_{\omega}\left(x_{n}(\omega)\right)=\int_{G_{n}} x_{n}(\omega) d \omega+\int_{I \backslash G_{n}} x_{n}(\omega) d \omega \leq x_{\left\lfloor K_{0}\right\rfloor}^{\alpha}+\frac{1}{2} \operatorname{Pr}\left(I \backslash G_{n}\right)$

where we have used Lemma 4.5 for the first term and the fact that $x_{n}(\omega) \leq$ $\frac{1}{2}$ for the second term. Now $x_{\left\lfloor K_{0}\right\rfloor}^{\alpha} \leq C_{1}\left(K_{0}^{-\frac{1}{\alpha}}\right) \leq C_{2}\left(n^{-\frac{1}{\alpha}}\right)$ when $K_{0}=p_{0} n$. On the other hand, the second term tends to zero exponentially fast. Since $x_{n}(\omega) \geq x_{n}^{\alpha} \geq C_{3} n^{-\frac{1}{\alpha}}$ by Lemma 4.4 and the fact that $x_{n}^{\alpha} \sim n^{-\frac{1}{\alpha}}$ we have established the required estimate on the expectation.

(2) This follows from part (1) immediately, since both maps have the same linear second branch.

(3) We have to get an estimate on

$$
(m \times m)\{\hat{R}>n\}=\sum_{l \geq n+1}(m \times m)\left(\Delta_{l}\right)=\sum_{l \geq n+1} \sum_{i \geq l+1}(m \times m)\left(\Delta_{0, i}\right) .
$$

First, observe that

$$
\begin{aligned}
(m \times m)\left(\Delta_{0, i}\right) & =\sum_{j=1}^{2^{i}}(m \times m)\left(\Delta_{0, i}^{j}\right)=\int_{0}^{1} J_{i}(\omega) d \omega \\
& =\int_{0}^{1}\left[x_{i-1}^{\prime}(\omega)-x_{i}^{\prime}(\omega)\right] d \omega \\
& =E\left[x_{i-1}^{\prime}(\omega)\right]-E\left[x_{i}^{\prime}(\omega)\right] \\
& =\frac{1}{2}\left[E\left(x_{i-1}(\omega)\right)-E\left(x_{i}(\omega)\right)\right] .
\end{aligned}
$$

Therefore, by equation (4.2) and part (1) of this proposition we have

$$
\begin{aligned}
(m \times m)\{\hat{R}>n\} & =\frac{1}{2} \sum_{l \geq n+1} \sum_{i \geq l+1}\left[E\left(x_{i-1}(\omega)\right)-E\left(x_{i}(\omega)\right)\right] \\
& =\frac{1}{2} \cdot\left[\left(E x_{n+1}(\omega)-E x_{n+2}(\omega)\right)+2\left(E x_{n+2}(\omega)-E x_{n+3}(\omega)\right)\right. \\
& \left.+3\left(E x_{n+3}(\omega)-E x_{n+4}(\omega)\right)+\ldots\right] \\
& =\frac{1}{2} \cdot\left[E\left(x_{n+1}(\omega)\right)+E\left(x_{n+2}(\omega)\right)+E\left(x_{n+3}(\omega)\right)+\ldots\right] \\
& \sim \sum_{k>n} k^{-\frac{1}{\alpha}} \\
& \sim n^{1-\frac{1}{\alpha}}
\end{aligned}
$$




\subsection{Distortion.}

Lemma 4.7. If $(x, \cdot),(y, \cdot) \in \Delta_{0}$ and $s((x, \cdot),(y, \cdot))=n$, then $|x-y| \leq \theta^{n}$.

Proof. Set $\theta:=\frac{1}{2}<1$ and observe that on $\Delta_{0}, D T_{\omega}^{R} \geq 2=\theta^{-1}$. Thus, if $(x, \cdot),(y, \cdot)$ lie in a common atom $\Delta_{0, i}^{j}$ such that $x, y \in J_{i}(\omega) \subseteq\left(T_{\omega}^{R}\right)^{-1}\left(\frac{1}{2}, 1\right]$, then

$$
\min D T_{\omega}^{R} \leq\left|\frac{T_{\omega}^{R} x-T_{\omega}^{R} y}{x-y}\right| \leq \frac{1}{|x-y|} .
$$

Therefore, $|x-y| \leq \theta$ and the result follows by induction on $k \leq n$.

Lemma 4.8. There exists a constant $C>0$ such that for $(x, \cdot),(y, \cdot) \in \Delta_{0, i}^{j}$,

$$
\left|\log \frac{D T_{\omega}^{R}(x)}{D T_{\omega}^{R}(y)}\right| \leq C\left|T_{\omega}^{R}(x)-T_{\omega}^{R}(y)\right| \leq C .
$$

Proof. It is trivial for $J_{i}, i=1$ since $T_{\alpha(\omega)}(x)=2 x-1$. We apply the Koebe principle to prove the result for $J_{i}, i \geq 2$.

Recall the Schwarzian derivative of a function $f \in C^{3}$ is given by:

$$
(\mathbf{S} f)(x)=\frac{f^{\prime \prime \prime}(x)}{f^{\prime}(x)}-\frac{3}{2}\left(\frac{f^{\prime \prime}(x)}{f^{\prime}(x)}\right)^{2} .
$$

It is also well known that the Schwarzian derivative of the composition of two functions $h, f \in C^{3}$ satisfies

$$
\mathbf{S}(h \circ f)(x)=(\mathbf{S} h)(f(x)) \times\left(f^{\prime}(x)\right)^{2}+(\mathbf{S} f)(x) .
$$

Consequently, Schwarzian derivative of the composition is negative if both functions have negative Schwarzian derivatives. Let $g$ denote the composition of the left branches of $T_{\alpha\left(\varphi^{R-1} \omega\right)}, \ldots, T_{\alpha(\varphi \omega)}$ and the right branch of $T_{\alpha(\omega)}$. Notice that on $J_{i}(\omega), i \geq 2$, we have $g(x)=T_{\omega}^{R}(x)$. Since $0<\alpha<\beta \leq 1$, we have for the left branch $T_{\alpha(\omega)}^{\prime}>0, T_{\alpha(\omega)}^{\prime \prime}>0$ and $T_{\alpha(\omega)}^{\prime \prime \prime} \leq 0$; in particular, $T_{\alpha(\omega)}^{\prime \prime \prime}=0$ if and only if $\alpha(\omega)=\beta=1$. Thus, $\mathbf{S} g<0$.

For each $J_{i}(\omega), i \geq 2$, let $J=\left[x_{i+1}^{\prime}(\omega), 2\right]$. Note that $g\left(x_{i+1}^{\prime}(\omega)\right)<\frac{1}{2}$. Set $\kappa:=$ $\frac{1}{2}-\sup _{\omega} g\left(x_{i+1}^{\prime}(\omega)\right)>0$. Then $J_{i}(\omega) \subset J$ and $g\left(J_{i}(\omega)\right)=\left(\frac{1}{2}, 1\right] \subset\left(\frac{1}{2}-\kappa, 2\right] \subset g(J)$. This means $g(J)$ contains a $\kappa$-scaled neighborhood of $g\left(J_{i}(\omega)\right)$ with constant $\kappa$. Therefore, by Koebe principle [9] there exists a constant $C(\kappa)>0$ such that

$$
\left|\log \frac{g^{\prime}(x)}{g^{\prime}(y)}\right| \leq C(\kappa) \frac{|x-y|}{\left|J_{i}(\omega)\right|} \leq C(\kappa), \quad \forall x, y \in J_{i}(\omega)
$$

and consequently,

$$
\left|\frac{g^{\prime}(x)}{g^{\prime}(y)}\right| \leq e^{C(\kappa)}
$$

It follows that

$$
\frac{|x-y|}{\left|J_{i}(\omega)\right|} \leq e^{C(\kappa)} \cdot \frac{|g(x)-g(y)|}{\left|g\left(J_{i}(\omega)\right)\right|} .
$$

Hence, $\left|\log \frac{g^{\prime}(x)}{g^{\prime}(y)}\right| \leq C(\kappa) \cdot e^{C(\kappa)} \cdot \frac{|g(x)-g(y)|}{\left|g\left(J_{i}(\omega)\right)\right|}$, which completes the proof.

Remark 4.9. Note that for $\beta>1,\left(\mathbf{S} T_{\beta}(x)\right)>0$ for $x \in\left(0, \frac{1}{2}\left(\frac{\beta-1}{(1+\beta)\left(1+\frac{\beta}{2}\right)}\right)^{1 / \beta}\right)$. 
Proposition 4.10. There exists a constant $C(F)>0$ such that for $z_{1}, z_{2} \in \Delta_{0, i}^{j}$,

$$
\left|\frac{D F^{R}\left(z_{1}\right)}{D F^{R}\left(z_{2}\right)}-1\right| \leq C(F) \cdot \theta^{s\left(F^{R}\left(z_{1}\right), F^{R}\left(z_{2}\right)\right)} .
$$

Proof. Let $z_{1}=\left(x_{1}, \omega_{1}\right), z_{2}=\left(x_{2}, \omega_{2}\right) \in \Delta_{0, i}^{j}$. Then they have same realization

$$
\left(\alpha\left(\omega_{l}\right), \alpha\left(\varphi \omega_{l}\right), \ldots, \alpha\left(\varphi^{R-1} \omega_{l}\right)\right),
$$

for $j=1,2$. Using this fact and $F^{R}\left(z_{l}\right)=S^{R}\left(z_{l}\right)$, for $z_{l} \in \Delta_{0, i}, l=1,2$, we have:

$$
\begin{aligned}
\frac{D F^{R}\left(z_{1}\right)}{D F^{R}\left(z_{2}\right)} & =\frac{D S^{R}\left(x_{1}, \omega_{1}\right)}{D S^{R}\left(x_{2}, \omega_{2}\right)} \\
& =\frac{\left|\begin{array}{cc}
D T_{\omega_{1}}^{R}\left(x_{1}\right) & \frac{\partial T_{\omega}^{R}}{\partial \omega^{2}}\left(z_{1}\right) \\
0 & D \varphi^{R}\left(\omega_{1}\right)
\end{array}\right|}{\left|\begin{array}{cc}
D T_{\omega_{2}}^{R}\left(x_{2}\right) & \frac{\partial T_{\omega}^{R}}{\partial \omega^{2}}\left(z_{2}\right) \\
0 & D \varphi^{R}\left(\omega_{2}\right)
\end{array}\right|} \\
& =\frac{D T_{\omega_{1}}^{R}\left(x_{1}\right) \cdot \frac{1}{P_{\omega_{1}}^{R}}}{D T_{\omega_{2}}^{R}\left(x_{2}\right) \cdot \frac{1}{P_{\omega_{2}}^{R}}} \\
& =\frac{D T_{\omega_{1}}^{R}\left(x_{1}\right)}{D T_{\omega_{2}}^{R}\left(x_{2}\right)}=\frac{D T_{\omega}^{R}\left(x_{1}\right)}{D T_{\omega}^{R}\left(x_{2}\right)},
\end{aligned}
$$

for any $\omega \in \Delta_{0, i}^{j}$. By using Lemma 4.7, Lemma 4.8 and the following inequality:

$$
|x-1| \leq \frac{e^{C}-1}{C}|\log x|, \text { if }|\log x| \leq C
$$

we obtain

$$
\begin{aligned}
\left|\frac{D T_{\omega}^{R}\left(x_{1}\right)}{D T_{\omega}^{R}\left(x_{2}\right)}-1\right| & \leq \frac{e^{C}-1}{C}\left|\log \frac{D T_{\omega}^{R}\left(x_{1}\right)}{D T_{\omega}^{R}\left(x_{2}\right)}\right| \\
& \leq \frac{e^{C}-1}{C} \times C\left|T_{\omega}^{R}(x)-T_{\omega}^{R}(y)\right| \\
& \leq C(F) \cdot \theta^{s\left(F^{R}\left(z_{1}\right), F^{R}\left(z_{2}\right)\right)} .
\end{aligned}
$$

\section{REFERENCES}

1. Ayyer, A., Liverani, C., Stenlund, M. Quenched CLT for random toral autormorphisms. Discr. and Cont. Dynam. Syst. 24 \#2 (2009), 331-348.

2. Bahsoun, W., Bose, C., Quas, A., Deterministic representation for position dependent random maps. Discrete Contin. Dyn. Syst. 22 (2008), 529-540.

3. Feller, W., Introduction to probability theory and its applications, Vol 2. John Wiley and Sons (1971).

4. Gouëzel, S., Sharp polynomial estimates for the decay of correlations. Israel J. Math. 139 (2004), 29-65.

5. Gouëzel, S., Statistical properties of a skew product with a curve of neutral points. Ergodic Theory Dynam. Systems 27 (2007), 123-151.

6. Hoeffding, W., Probability inequalities for sums of bounded random variables J. Amer. Stat. Soc. 58 \# 301 (1963), 13-30.

7. Hu, H., Decay of correlations for piecewise smooth maps with indifferent fixed points. Ergodic Theory Dynam. Systems 24 (2004), 495-524. 
8. Liverani, C., Saussol, B. and Vaienti, S., A probabilistic approach to intermittency, Ergodic Theory Dynam. Systems 19 (1999), 671-685.

9. de Melo, W., van Strien, S. One-dimensional dynamics. Springer-Verlag, Berlin, 1993.

10. Melbourne, I., Terhesiu, D. Operator renewal theory and mixing rates for dynamical systems with infinite measure. Invent. Math. 189 (2012), no. 1, 61-110.

11. Pène, F., Averaging method for differential equations perturbed by dynamical systems. ESAIM Probab. Statist. 6 (2002), 33-88.

12. Pianigiani, G. First return map and invariant measures. Israel J. Math. 35 (1980), 32-48.

13. Thaler, M., Estimates of the invariant densities of endomorphisms with indifferent fixed points. Israel J. Math., 37 (1980), 303-314.

14. Young, L-S., Recurrence times and rates of mixing. Israel J. Math., 110 (1999), 153-188.

Department of Mathematical Sciences, Loughborough University, Loughborough, LeICESTERSHIRE, LE11 3TU, UK

E-mail address: † W.Bahsoun@lboro.ac.uk, $\ddagger$ Y.Duan@lboro.ac.uk

Department of Mathematics and Statistics, University of Victoria, PO BOX 3045 Stn

CSC, Victoria, B.C., V8W 3R4, Canada

E-mail address: * cbose@uvic.ca 